\title{
It is time for a harmonized ethical review procedure across Europe
}

\author{
Pieter J. Hoekstra $\cdot$ Jan K. Buitelaar
}

Published online: 8 September 2013

(C) Springer-Verlag Berlin Heidelberg 2013

This month's issue of European Child and Adolescent Psychiatry contains a nice set of well-conducted studies involving children and adolescents as research subjects. Isolan et al. (REF) studied children and adolescents from the general population in Brazil and found that being a victim of bullying is clearly associated with more anxiety symptoms. While this being a single time point observational study made it not possible to demonstrate causality, the authors rightly argue that the relationship between being bullied and symptoms of anxiety most probably is bidirectional in nature. On one hand being bullied is a considerable source of distress, which may increase anxiety symptoms; conversely, anxiety and anxious behavior could make a child appear more nervous and uncomfortable with peers and thus more vulnerable to aggressive peers and make him a possible target of further victimization. Another study in this issue, from the Netherlands, addressed the cost-effectiveness of different screening strategies to prevent anxiety (Simon REF this issue). Screening and offering a parent-focused intervention to children of anxious parents, and a child-focused intervention to children of non-anxious parents, was found to be the most cost-effective approach. The study has clear implications for policy makers.

\section{P. J. Hoekstra ( $\square)$}

Department of Psychiatry, University Medical Center

Groningen, University of Groningen, Groningen,

The Netherlands

e-mail: p.hoekstra@accare.nl

\section{J. K. Buitelaar}

Department of Cognitive Neuroscience, Radboud University

Nijmegen Medical Center, and Karakter Child and Adolescent Psychiatry, Nijmegen, The Netherlands
In perhaps the most intriguing study of the current issue, Schmitz and colleagues (REF this issue) investigated a range of autonomic measurements in three different domains (cardiac, vascular, and electrodermal sympathetic activation) prior to, during, and after a social stress task in a sample of high and low socially anxious. The high socially anxious children showed blunted cardiac response to the social stress task pointing to restricted cardiac flexibility in these children. The authors conclude that this demonstrates that social anxiety cannot be considered a mere cognitive phenomenon, which has clear consequences of our understanding and best treatment of this impairing condition.

When reading the article of Schmitz et al., few readers if any will pay much attention to an almost ubiquitous single sentence within the paper's methods section: The current study was approved by the local ethics committee. The amount of work and time behind this simple sentence, however, is not to be underestimated, especially when it comes to non-commercial clinical trials. For example, Hackshaw et al. [1] demonstrated that it took more than half of the UK sites involved in a multicenter trial more than 6 months to get full ethical approval for a relatively straightforward low-risk treatment study, with delays of 12 months or more until approval being quite common. Whereas already the process of designing a study, obtaining agreement for drug supply from the pharmaceutical industry, and submitting the trial for funding can rarely be achieved in less than 12-18 months, a further delay of almost a year to recruit the first patient could clearly make Europe less attractive for the conduct of clinical trials. Hackshaw et al. [1] point out that such a delay not only extends the duration of a trial, but also increases its cost. Employing a trial coordinator for an extra year might cost about $€ 50,000$, so for every ten trials that need to be 
extended by a year the increase in cost is $€ 500,000$. This could fund at least one new trial.

When setting up a non-commercial multi-center study across various different countries, e.g., in the context of funding by the European commission, the situation is even more complex and burdensome. Although the European Union has set a Directive for a better harmonization of the ethical review practice between the various member states, many problems remain, as still approval from each separate national or local ethical committee is to be obtained and precise requirements for content, liability insurance conditions, site assessment, and consent procedures vary from country to country, thus jeopardizing the existence of a uniform research protocol. Moreover, although a single declaration of Helsinki forms the base of all ethical committees, variations in judgments between different ethical committees on one and the same research protocol can be substantial and the various protocol change requests by ethical committees also often seriously challenge the maintenance of a uniform protocol across all sites. Our experience is that there are country-specific patterns in ethical review policies. For example, in the Netherlands, much emphasis is put on reviewing design and methodology aspects of a research protocol, sometimes not unlike a grant review process, whereas in the UK feasibility of the project and degree of burden for participants are much more the focus of the ethical review. Other country-specific policies have an undesirable direct impact on the type of research that is at all possible in a country. A striking example is that brain magnetic resonance imaging (MRI) for research purposes is not allowed in children below the age of 6 in the Netherlands, in contrast to the UK and the USA where scanning of newborns is well accepted (e.g., [2]).

The USA does not have a central review process either. Silberman and Kahn [3] have reviewed the practice of institutional review boards within the USA and concluded that different review boards that were presented with identical protocols sometimes asked for different and even competing revisions. Another study [4] also had found that review of a single uniform protocol for a multicenter genetic epidemiology study by local review boards within the USA was highly variable, with lack of uniformity in the review process creating uneven human subjects protection and challenging a uniform protocol.

The European Commission is currently considering the possibility to revise the clinical trials legislation. A 2010 Multidisciplinary Workshop on Research Ethics Committees and Ethical Review in Europe came with the recommendation to further explore the possibility for a single ethics review opinion on European Union level for multinational clinical trials (see http://www.efgcp.be). In a globalized world and in the context of large scale public funding for studies across Europe or beyond, such a streamlined ethical review procedure is certainly to be warmly endorsed, in the interest of effectively using public funds while uniformingly protecting the rights and health of the children and adolescents who we approach for our studies.

\section{References}

1. Hackshaw A, Farrant H, Bulley S, Seckl MJ, Ledermann JA (2008) Setting up non-commercial clinical trials takes too long in the UK: findings from a prospective study. J R Soc Med 101(6):299-304. doi:10.1258/jrsm.2008.070373

2. Toews M, Wells WM 3rd, Zöllei L (2012) A feature-based developmental model of the infant brain in structural MRI. Med Image Comput Comput Assist Interv 15(Pt 2):204-211

3. Silberman G, Kahn KL (2011) Burdens on research imposed by institutional review boards: the state of the evidence and its implications for regulatory reform. Milbank Q 89(4):599-627

4. McWilliams R, Hoover-Fong J, Hamosh A, Beck S, Beaty T, Cutting G (2003) Problematic variation in local institutional review of a multicenter genetic epidemiology study. JAMA 290(3):360-366 